\title{
Myalgia, CTCAE
}

National Cancer Institute

\section{Source}

National Cancer Institute. Myalgia, CT CAE. NCI Thesaurus. Code C143688.

A disorder characterized by marked discomfort sensation originating from a muscle or group of muscles. 